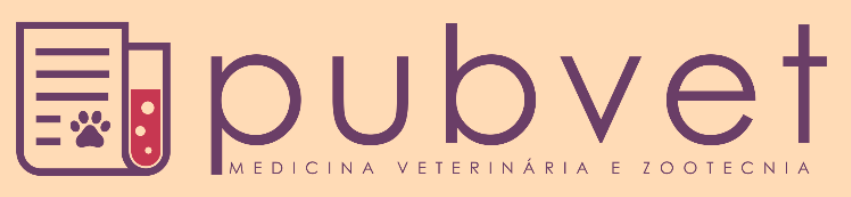

https://doi.org/10.31533/pubvet.v14n11a693.1-12

\title{
Estratégias de manejo do pastejo para produção intensiva de leite em pastos tropicais
}

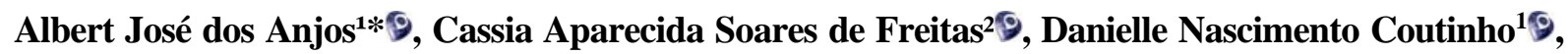 \\ Bárbara Cardoso da Mata e Silva ${ }^{39}$, Haviner Paixão de Sena ${ }^{4}$, Bruno Bernardino Soares ${ }^{4}$, \\ Carolina de Paula Pires 5 , Rafael Lelis de Freitas $5 \sqrt{9}$ \\ ${ }^{I}$ Doutorando(a) em Zootecnia, Universidade Federal de Viçosa, Departamento de Zootecnia. Viçosa - MG Brasil. \\ ${ }^{2}$ Doutora em Zootecnia, Universidade Federal de Viçosa, Departamento de Zootecnia. Viçosa - MG Brasil. \\ ${ }^{3}$ Doutora em Zootecnia, Universidade Federal de Minas Gerais, Departamento de Zootecnia. Belo Horizonte - MG Brasil. \\ ${ }^{4}$ Graduando em Agronomia, Universidade Federal de Viçosa, Departamento de Zootecnia. Viçosa - MG Brasil. \\ ${ }^{5}$ Graduando(a) em Zootecnia, Universidade Federal de Viçosa, Departamento de Zootecnia. Viçosa - MG Brasil. \\ *Autor para correspondência, E-mail: albert@zootecnista.com.br
}

Resumo. A intensificação do sistema de produção de leite a pasto é prática necessária, uma vez que além de aumentar a produtividade por área torna possível a disponibilização de áreas para outras atividades agrícolas. O período de descanso variável, respeitando as características morfofisiológicas da planta, tem substituído o período de descanso fixo, tradicionalmente utilizado em sistemas de pastejo rotacionado por décadas. Períodos de descanso com base no desenvolvimento morfofisiológico da planta tem proporcionado a produção de maior quantidade de forragem, constituída principalmente por maior percentual de folhas, o que tem garantido melhor valor nutritivo a forragem ingerida. Aliado a adequada intensidade de desfolha este método de manejo tem possibilitado a obtenção de maior produtividade no sistema de produção de leite a pasto, possibilitando a melhoria dos índices econômicos da atividade.

Palavras-chave: altura, consumo, interceptação luminosa, intervalo entre pastejos, período de ocupação

\section{Grazing management strategies for intensive milk production in tropical pastures}

Abstract. The intensification of the pasture milk production system is a necessary practice, since in addition to increasing productivity by area, it is possible to make areas available for other agricultural activities. The variable rest period, respecting the plant's morphophysiological characteristics, has replaced the fixed rest period, traditionally used in rotational grazing systems for decades. Rest periods based on the morphophysiological development of the plant have provided the production of greater amount of forage, consisting mainly of a higher percentage of leaves, which has guaranteed better nutritional value to the ingested forage. Combined with adequate defoliation intensity, this management method has made it possible to obtain greater productivity in the pasture milk production system, enabling the improvement of economic activity rates.

Keywords: consumption, height, interval between defoliation, luminous interception, period of occupation

\section{Estrategias de manejo del pastoreo para la producción intensiva de leche em pasturas tropicales}

Resumen. La intensificación del sistema de producción de leche de pasto es una práctica necesaria, ya que además de aumentar la productividad por área, es posible poner áreas 
disponibles para otras actividades agrícolas. El período de descanso variable, respetando las características morfofisiologías de la planta, ha reemplazado el período de descanso fijo, utilizado tradicionalmente en los sistemas de pastoreo rotativo durante décadas. Los períodos de descanso basados em el desarrollo morfofisiológico de la planta han proporcionado la producción de una mayor cantidad de forraje, constituida principalmente en un mayor porcentaje de hojas, lo que ha garantizado un mejor valor nutricional para el forraje ingerido. Combinado con una intensidad de defoliación adecuada, este método de gestión ha permitido obtener una mayor productividad em el sistema de producción de leche de pastoreo, permitiendo la mejora de las tasas de actividad económica.

Palabras clave: altura, consumo, intercepción de luz, intervalo de pastoreo, período de ocupación

\section{Introdução}

O manejo do pastejo tem se caracterizado como uma importante ferramenta de intensificação, visto que, pela adoção de práticas adequadas e de baixo custo, tem-se obtido acréscimos consideráveis na produção e produtividade (Pinto et al., 2001). No entanto, mesmo diante da grande relevância das áreas de pastos para a pecuária, observa-se que o sistema produtivo nacional tem-se caracterizado como um modelo de produção extrativista, onde a adoção de tecnologias e o uso intensivo em capital têm sido adotados por uma pequena parcela de produtores (Barcellos et al., 2008), refletindo a baixa eficiência produtiva dos sistemas tropicais de produção de leite.

De forma geral, sistemas intensivos de produção de leite a pasto são caracterizados pela utilização do método de pastejo sob lotação rotativa (Pompeu et al., 2009). Esse sistema é caracterizado pela subdivisão da área total da pastagem em um número variável de piquetes menores, que são pastejados pelos animais por um determinado período de tempo (período de ocupação), sendo posteriormente submetido a um período de descanso, quando os animais são então retirados da área (Euclides \& Barbosa, 2008). A determinação do período de ocupação e do período de descanso pode sofrer uma série de combinações, e a esse processo denominamos de manejo do pastejo.

Embora a intensificação do sistema de produção de leite a pasto se caracterize como uma ferramenta estratégica para a maximização da taxa de lotação e da produção animal por área (Pereira \& Cóser, 2002) e mesmo diante do grande avanço ocorrido nas técnicas de manejo de pastagens e do pastejo nos últimos tempos, a falta de informação adequada principalmente sobre o manejo do pastejo têm gerado grandes prejuízos ao desenvolvimento da pecuária nacional.

Dessa forma, esta revisão tem como objetivo apresentar alternativas de manejo do pastejo que possam colaborar no processo de desenvolvimento de sistemas de produção de leite baseados no uso intensivo de gramíneas tropicais.

\section{Produção de leite a pasto}

No passado, os sistemas de produção de leite a pasto tinham como princípio a baixa utilização de insumos, a utilização de forrageiras menos exigentes em fertilidade e adaptadas às condições de solos ácidos ou tolerantes a toxidez por alumínio, fato que além de ocasionar baixos níveis de produtividade, elevava o custo de produção por quilograma de leite produzido (Silva et al., 2012). Um dos resultados desse processo foi o declínio produtivo das pastagens, e a consequente substituição das forrageiras já estabelecidas na tentativa de restabelecer a produtividade, dando início ao denominado "ciclo dos capins" (Fonseca et al., 2010). Diante desse contexto, tornaram-se necessárias à adoção de práticas de manejo que reduzissem ou não elevassem os custos de produção, além de possibilitarem o uso eficiente de recursos ambientais e econômicos, otimizando dessa forma a produtividade por animal e por área (Aurélio et al., 2007).

O sistema de produção intensiva de leite a pasto brasileiro é caracterizado por uma produtividade animal variando entre 2.000 a 4.500 litros por lactação (Assis et al., 2005). Isso equivale a uma produtividade média, variando entre 8 a 15 litros por animal dia, dependendo da raça utilizada e da utilização ou não de suplementação. Embora muito se diga sobre a produção por animal, a principal característica vislumbrada em sistemas de produção intensiva de leite a pasto é a produtividade por área. 
De maneira geral, a produtividade por área tem variado entre 75 a $137 \mathrm{~kg} / \mathrm{ha} / \mathrm{dia}$, dependendo do manejo utilizado (Moura et al., 2017; Santos et al., 2011).

A principal forma utilizada para intensificação da produção de leite a pasto é a utilização do método de pastejo sob lotação rotativa, sendo possível melhorar a qualidade da forragem fornecida aos animais (Santos et al., 2008). Este sistema tem como principal característica a exploração de forrageiras de alta produtividade, durante o período das águas (Euclides \& Barbosa, 2008) tornando possível o aumento da capacidade de suporte do pasto, o aumento da produção por animal e da produtividade por área, e economia no uso de concentrados (Pereira \& Cóser, 2002).

Inicialmente, mais especificamente nos anos 80 , a intensificação da produção leiteira a pasto foi baseada na utilização do capim-elefante, sendo que posteriormente gêneros como Cynodon, Megathyrsus e Uruchloa passaram a ser utilizados (Lopes et al., 2017). A opção por tais gêneros devese principalmente a suas características produtivas, evidenciadas pela elevada capacidade produtiva de matéria seca e alta proporção de folhas (Porto et al., 2009). Como esta revisão tem como objetivo discutir técnicas de manejo do pastejo para a produção intensiva de leite a pasto o enfoque será destinado somente àquelas forrageiras utilizadas no sistema intensivo de produção.

O processo de melhoramento genético do rebanho tem passado por grandes transformações nos últimos tempos e técnicas como a fertilização in vitro, transferência de embriões e inseminação artificial em alinhamento com a seleção genética tem contribuído de forma maciça para a melhoria da produtividade leiteira nacional (Rigolon et al., 2003). Entretanto, conforme mencionado por Fariña et al. (2011), o mérito genético dos animais por si só, não resolve os gargalos produtivos da atividade leiteira, já que o manejo tem reflexos mais acentuados sobre a produtividade do que o melhoramento genético.

No contexto alimentar, o manejo do pastejo apresenta importância primordial, podendo afetar a composição morfológica pela produção de folhas, colmos e material morto, e consequentemente a estrutura do dossel, determinando o comportamento ingestivo dos animais e o consumo de nutrientes (Reis et al., 2009). O correto emprego dos termos manejo da pastagem e manejo do pastejo necessita ser previamente mencionado, uma vez que confusões com a utilização de ambos os termos é comumente visualizada no meio técnico. Manejo da pastagem refere-se à manipulação do sistema solo-planta-animal em áreas de pasto, visando objetivos específicos (e.g. adubação e correção do solo, irrigação, controle de pragas, subdivisão de áreas), enquanto o termo manejo do pastejo é corretamente empregado ao se mencionar a remoção de forragem, pela atividade de pastejo, podendo esta ser manipulada através da intensidade e frequência de desfolha (Pinto \& Ávila, 2013).

Toda planta forrageira apresenta uma condição estrutural ideal, do ponto de vista a garantir o maior consumo de forragem, o que torna necessário o profundo conhecimento das variáveis estruturais do dossel forrageiro, de forma que o manejo do pastejo leve em consideração os eventos fisiológicos (e.g. crescimento, senescência, alongamento de colmo) que ocorrem durante todo o ciclo de desenvolvimento da planta forrageira (Lima \& Rodriguez, 2009). Práticas de manejo devem ser programadas de forma a respeitar uma escala hierárquica, onde os limites de produção da planta forrageira (interface solo-plantameio) devem ser primariamente considerados, para que posteriormente o componente animal possa ser assinalado como fonte produtora de leite (Euclides \& Barbosa, 2008). Entretanto, o principal objetivo das estratégias de manejo, sejam elas do pastejo (e.g. intervalo entre desfolhas, intensidade de desfolha, período de ocupação) ou da pastagem (e.g. adubação), devem potencializar o desempenho dos animais e aperfeiçoar a utilização dos recursos forrageiros basais (Reis et al., 2012).

\section{Características estruturais do dossel}

Estratégias de manejo do pastejo, tais como o pastejo contínuo com alta taxa de lotação e o pastejo rotativo com reduzido período de descanso devem buscar formas de controle das características estruturais do dossel, de forma a prevenir altas taxas de alongamento de colmo, que apesar de promoverem maior taxa de acúmulo de forragem, comprometem a composição morfológica do relvado acarretando em redução do consumo animal (Euclides et al., 2008).

As características estruturais do dossel são afetadas pelos diversos processos fisiológicos observados durante a produção primária (fotossíntese, respiração, perfilhamento, expansão e senescência foliar), de 
forma que essas características são diretamente influenciadas pelo período de descanso utilizado (Teixeira \& Oliveira, 2009). Dentre os constituintes da massa de forragem, especial atenção deve ser destinada às lâminas foliares, uma vez que estas compõem mais de $80 \%$ da forragem consumida pelos animais (Euclides \& Barbosa, 2008). A composição morfológica também pode ser influenciada pelo ajuste da taxa de lotação, já que condições de sub ou super pastejo ao longo do ano podem modificar o consumo de forragem por parte dos animais e assim comprometerem a estrutura do relvado (Euclides \& Barbosa, 2008).

\section{Valor nutritivo}

A prevalência de gramíneas forrageiras do tipo $\mathrm{C} 4$, forrageiras com alta eficiência fotossintética e acelerada velocidade de crescimento, em conjunto com as variáveis climáticas e o inadequado manejo do pastejo são os principais fatores responsáveis pela rápida perda do valor nutritivo das forrageiras tropicais (Lopes, 2008). A ocorrência de desequilíbrios oriundos da adoção de técnicas de manejo inadequadas pode trazer consequências na produção e valor nutritivo da forragem, com influência na composição química e digestibilidade da planta (Cecato et al., 2000).

No caso da produção intensiva de leite a pasto, o melhor valor nutritivo pode ser evidenciado principalmente pela maior presença de componentes morfológicos de melhor digestibilidade, como é o caso das folhas, em relação aqueles de menor digestibilidade. Conforme Paciullo et al. (2002), existe uma diferença na composição química e física de cada tecido da planta, e esta diferença está relacionada principalmente a função que cada órgão desempenha. Dessa forma, tecidos destinados à sustentação da planta, como no caso dos colmos, possuem células densamente agrupadas, com paredes espessas e lignificadas, diferentemente de tecidos como as folhas, que são tecidos destinados ao processo de assimilação de carbonos (fotossíntese), sendo ricos em cloroplastos, e apresentando células com parede delgada e não lignificada (Paciullo et al., 2002). Além disso, a baixa digestibilidade e o baixo consumo das forrageiras tropicais também se associam ao seu reduzido conteúdo de proteínas e minerais, e ao alto conteúdo de fibra (Euclides \& Barbosa, 2008).

\section{Oferta de forragem e consumo de forragem}

Em trabalho de revisão de literatura, Gomide (1998) sugeriu que em pastos manejados sob lotação rotativa, níveis de oferta de forragem variando entre 5 a $7,5 \mathrm{~kg}$ de massa seca verde para cada $100 \mathrm{~kg}$ de peso vivo permitiriam maximizar a produção de leite por vaca. Todavia, sistemas submetidos a níveis de oferta maiores que $10 \mathrm{~kg}$ de forragem $/ 100 \mathrm{~kg}$ de peso vivo correspondem a perdas de forragem maiores que 50\%, caracterizando um baixo aproveitamento de forragem (Braga et al., 2007). A adoção de práticas de manejo do pastejo capazes de ofertar forragem de boa qualidade, e em quantidades que não limitem o consumo animal tornam-se fatores-chave para obtenção de bons resultados na produção intensiva de leite a pasto. Assim, atenção deve ser destinada a este ponto, uma vez que a alta oferta de forragem apesar de propiciar alta produção por animal, uma vez que o animal tem oportunidade para selecionar a forragem, optando sempre por aquela de melhor qualidade (e.g. folhas em detrimento de colmo), resulta em menor eficiência de pastejo, acarretando notoriamente em baixa produtividade por área. Entretanto, o aumento na pressão de pastejo, de forma a não causar grandes prejuízos ao consumo animal poderá resultar no incremento da produção por área (igigura 1).

$\mathrm{O}$ aperfeiçoamento de práticas de manejo do pasto e do animal visando maximizar o consumo de forragem e a produção de leite tem sido um dos principais desafios imposto aos pesquisadores, consultores e produtores de leite (Santos et al., 2008). Uma vez que a estrutura do pasto exerce influência no comportamento ingestivo dos animais, a adoção de estratégias de manutenção da estrutura do pasto capaz de manter alta quantidade de folhas no dossel forrageiro, podem otimizar a colheita de forragem pelo animal (Aurélio et al., 2007). Como 90\% dos colmos são encontrados na metade inferior do dossel forrageiro, intensidades de desfolha que não ultrapassem 50\% da altura inicial do dossel, podem propiciar melhores condições de consumo, uma vez que o material colhido será composto por pequena quantidade de colmos (Porto et al., 2009). Juntamente com a intensidade de desfolha características como altura da planta, população de perfilhos, densidade de biomassa, relação folha/colmo, proporção de material morto e presença de inflorescência também podem condicionar o consumo (Porto et al., $\underline{2009)}$. 


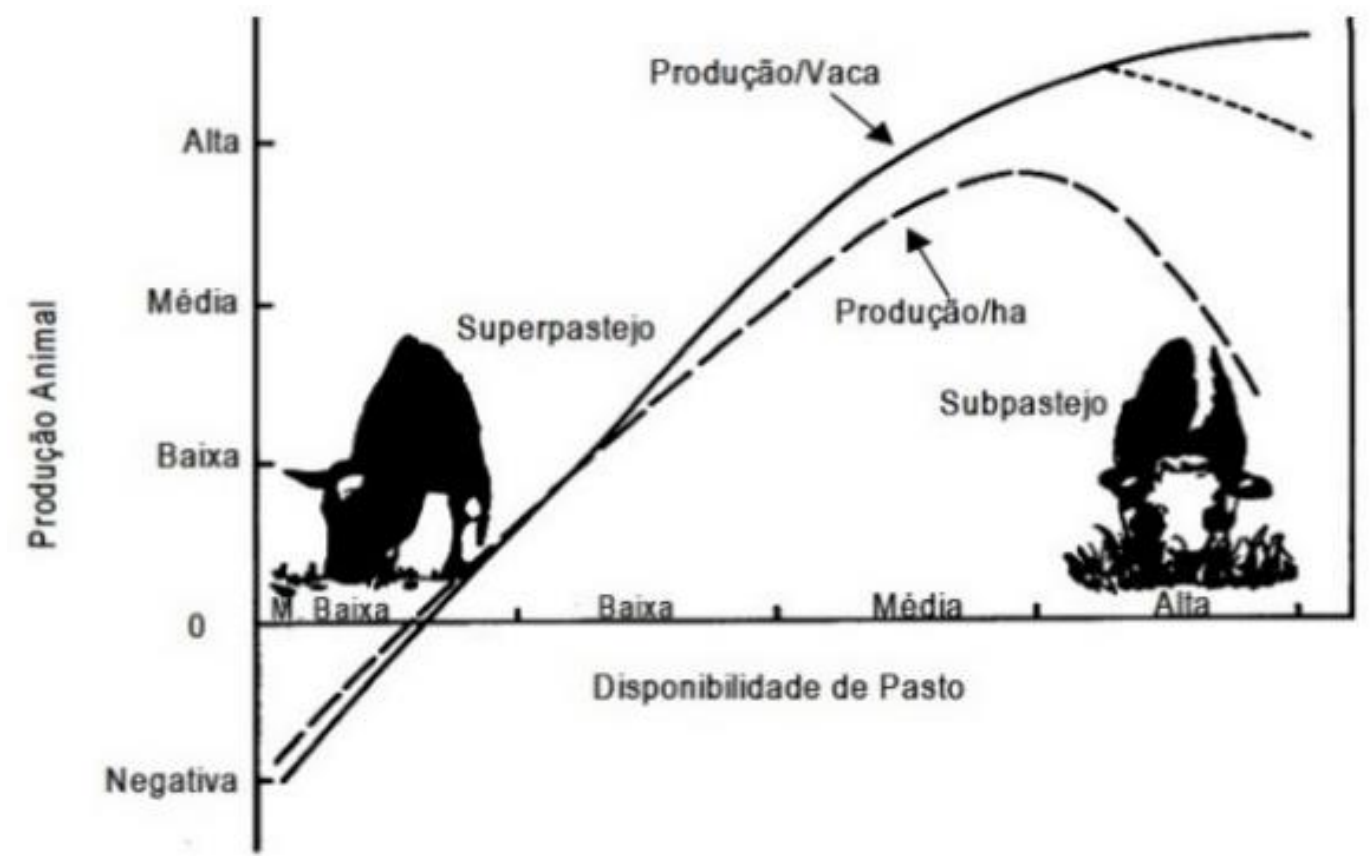

Figura 1. Relação entre a disponibilidade de pasto e a produção por vaca e por hectare. Fonte: Blaser (1988).

\section{Intervalo entre pastejo}

Os princípios básicos de produção primária da planta (fotossíntese, respiração, perfilhamento, expansão e senescência foliar), juntamente com fatores ambientais (água, temperatura, luminosidade) e com a intensidade de utilização devem ser levados em consideração na determinação do período de descanso (Euclides \& Barbosa, 2008). Por um longo período, a utilização do período de descanso baseado em dias fixos foi prática rotineira em sistemas de produção intensiva de leite a pasto. A utilização de tal padrão ocorreu principalmente pela associação entre idade e maturidade, fato que levou a aplicação da idade de corte ou pastejo como sendo um critério para a qualidade de plantas forrageiras (Van Soest, 1994). Todavia, pesquisas desenvolvidas com o capim-xaraés (Pedreira et al., 2009); capimelefante (Voltolini et al., 2010) e capim-marandu (Anjos et al., 2016) evidenciaram a negatividade da adoção do período de descanso fixo em relação ao período de descanso variável conforme a interceptação de $95 \%$ da radiação fotossinteticamente ativa pelo dossel (IRFA 95 ).

Contrariamente, ao que foi por muito tempo pregado, a utilização do período de descanso fixo não é a prática mais adequada para sistemas de produção de excelência, uma vez que fatores abióticos como radiação solar, temperatura, água e nutrientes influenciam diretamente a produção de biomassa vegetal (Gomide et al., 2003). Assim, a utilização de períodos de descanso fixo poderia resultar em situações de super ou sub pastejo, afetando a eficiência produtiva do sistema (Cândido et al., 2006). Outro ponto importante é que o aumento na produção de massa seca não reflete necessariamente em produtividade animal, já que pastos submetidos a períodos de descanso prolongados (e.g. período de descanso maior que a IRFA ${ }_{95}$ ) apresentam maior perda por senescência, além de terem sua estrutura comprometida pelo maior alongamento de colmo (Corsi, 1985).

As plantas forrageiras são submetidas constantemente ao estresse da colheita e, diante disso, se faz necessário conhecer as habilidades de recuperação das plantas após o pastejo, levando em conta suas características fisiológicas e as condições do ambiente, pois somente dessa forma o manejo poderá ser totalmente eficiente, garantindo boa produtividade à planta (Lima \& Rodriguez, 2009). Condições de super pastejo devem ser evitadas, uma vez que a área foliar remanescente tem reflexos sobre a velocidade e vigor de rebrota (Fonseca et al., 1998). A adoção de níveis crescentes de adubo, principalmente o nitrogenado, torna possível o aumento da frequência de pastejo, caso as condições edafoclimáticas sejam favoráveis ao crescimento e não existam outros fatores limitantes (Euclides \& Barbosa, 2008). 
A adoção de períodos de descansos mais longos, sejam eles baseados em dias fixos ou superiores a IRFA $_{95}$, resulta no comprometimento da estrutura do dossel, fato derivado do elevado acúmulo de colmos e material morto observado nestas condições (Pedreira et al., 2009).

A manutenção do dossel forrageiro em alturas mais baixas, situação comum na época chuvosa ao se optar pelo manejo com base na IRFA ${ }_{95}$ em comparação ao período de descanso com dias fixos (Anjos et al., 2016), apesar de propiciar menor acúmulo e crescimento de forragem, também reduz sua senescência (Reis et al., 2009). Entretanto, pastos submetidos a maiores períodos de descanso podem não necessariamente resultar no maior acúmulo de forragem (Carnevalli et al., 2006). Isso é totalmente explicável, já que o crescimento da planta é condicionado a fatores ambientais, o que mais uma vez torna equivocado a utilização do período de descanso fixo.

Anjos et al. (2016) observaram maior massa seca de forragem na condição de pré-pastejo em pastos de Uruchloa brizantha cv. Marandu submetidos ao período de descanso fixo de 30 dias em comparação aqueles sob período de descanso variável, conforme a IRFA95 (com médias de 5.290 e $4.411 \mathrm{~kg}^{-1} \mathrm{ha}^{-1}$ ciclo $^{-1}$ de pastejo, respectivamente). Todavia, ao se avaliar a composição morfológica do dossel, os pastos submetidos ao período de descanso variável apresentaram maior percentual de folhas (48.3 vs. 41.1) e menor percentual de material morto (19.0 vs. 25.4) em relação ao pasto com período de descanso fixo. Maior proporção de folhas e menores proporções de colmo e material morto foram observadas em pastos submetidos ao menor período de descanso, caracterizado pela IRFA ${ }_{95}$, resultando em maior eficiência de pastejo (Carnevalli et al., 2006). Além disso, intervalos entre pastejo com base na IRFA 95 apesar de resultarem em menor massa de forragem por ciclo de pastejo, são capazes de propiciarem maior número de ciclos de pastejo por ano em comparação ao intervalo entre pastejo com período fixo, garantindo ao final do período maior produção de forragem (Voltolini et al., 2010).

\section{Intensidade de pastejo}

Embora maior eficiência de pastejo possa ser obtida em condições onde a intensidade de desfolha seja maior do que $50 \%$ da altura de entrada dos animais no piquete (Carnevalli et al., 2006), sistemas de produção intensiva de leite a pasto devem optar pela utilização de moderada intensidade de pastejo, visto que intensidades maiores que $50 \%$ da altura inicial resultam no comprometimento da estrutura do dossel, dificultando o acesso dos animas a forragem disponível, devido à elevada presença de colmos no estrato inferior do dossel, exceto em condições de alta frequência de pastejo (Porto et al., 2009). Outro ponto que merece especial atenção é com relação à dificuldade para a obtenção de intensidades de pastejo superiores a $50 \%$ da altura inicial em algumas situações, como por exemplo, no manejo do capimelefante (Schmitt et al., 2019).

A maior participação de colmos no estrato inferior de pastos de capim-aruana (Megathyrsus maximus cv. Aruana) submetidos à combinação entre duas frequências (95\% e 98\%) e duas intensidades de desfolhação (10 e $15 \mathrm{~cm}$ de altura pós-pastejo) foi demonstrada por Zanini et al. (2012). Os autores observaram que independentemente da frequência de desfolhação, $90 \%$ do total de colmos presentes no pasto encontrava-se na metade inferior do dossel.

\section{Período de ocupação dos piquetes}

A duração do período de ocupação dos piquetes é extremamente importante, visto que o balanço entre a quantidade e qualidade da forragem disponível tem reflexo direto sobre a produtividade animal (Cóser et al., 1999). Ao contrário de sistemas com baixo uso de fertilizantes, onde as taxas de acúmulo de forragem e lotação são baixas, possibilitando a adoção de períodos de ocupação mais longos, sistemas intensivos são caracterizados pela alta utilização de fertilizantes e pelo elevado número de animais por área, o que torna recomendável a adoção de períodos de ocupação reduzidos, restringindo dessa forma a oportunidade do animal consumir a forragem em rebrota (Martha Júnior, 2003). Embora o maior período de ocupação dos piquetes resulte na necessidade de menor número de piquetes, o que notoriamente acarreta em menor investimento em estruturas como cercas, bebedouro e maior facilidade para manejo dos animais (Fonseca et al., 1998) o menor período de ocupação torna menor a seletividade dos animais. 
Cóser et al. (1999) avaliaram o efeito de três diferentes períodos de ocupação (1, 3 e 5 dias) sobre a produção de leite/animal/dia de vacas mestiças Holandês x Zebu em pastos de capam-elefante (Pennisetum purpureum Schum. cv. Napier) por um período de três anos e observaram maior variação na produção diária nos animais mantidos nos piquetes com maior período de ocupação (Figura 2).

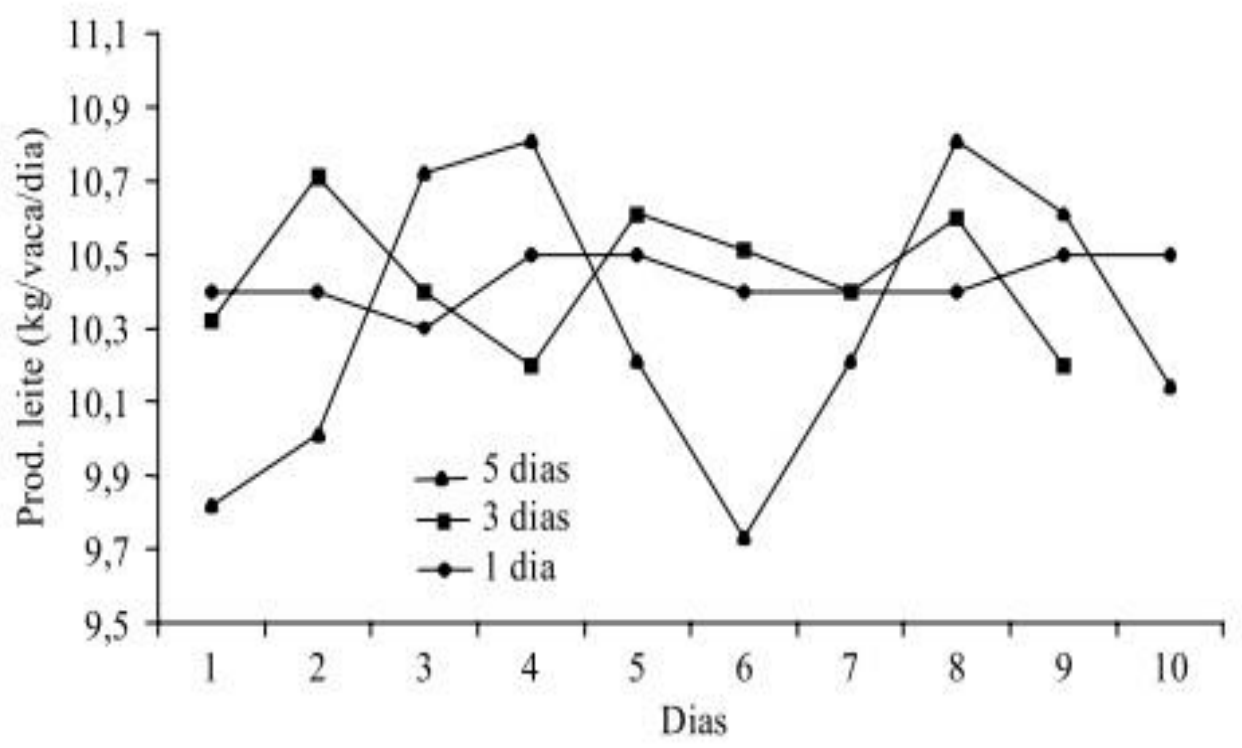

Figura 2. Variação diária na produção de leite de vacas mestiças Holandês x Zebu mantidas em pasto de capimelefante com período de ocupação de 1, 3 e 5 dias, durante um período de dez dias. Fonte: Cóser et al. (1999).

Araújo et al. (2020) observaram redução no consumo de matéria seca de 8,5\% e 36,9\% no segundo e terceiro dia de ocupação dos piquetes, para novilhas Holandês x Zebu mantidas em pastos de capimxaraés (Uruchloa brizantha cv. Xaraés), atribuindo essa redução à indisponibilidade de massa de forragem de qualidade, principalmente folhas, já que estas reduzem progressivamente sua participação durante o período de ocupação dos piquetes, uma vez que as lâminas foliares são primariamente consumidas. No capim-mombaça (Megathyrsus maximus Jacq. cv. Mombaça) decréscimos na qualidade nutritiva com o avanço no período de ocupação foram relacionados principalmente ao aumento de constituintes fibrosos (Clipes et al., 2006).

A variação na produção de leite nos diferentes dias de ocupação dos piquetes é justificada pelo consumo de forragem de menor qualidade à medida que o período de ocupação avança, sendo que, após serem colocadas em um novo piquete, esse efeito é então suprimido após um ou dois dias, já que o animal nos dias iniciais de pastejo tem acesso à forragem de melhor digestibilidade (Cóser et al., 1999). Entretanto, apesar da menor variação diária na produção de leite de vacas mantidas em pastos submetidos ao período de ocupação de um dia e do reflexo observado no valor nutritivo de algumas forrageiras submetidas ao período de ocupação de três dias, a adoção do período de ocupação de três dias pode conciliar a estabilidade produtiva e a uniformidade do pastejo com a necessidade de um reduzido número de piquetes (Gomide et al., 2012).

\section{Indicações de manejo}

Com base em uma compilação de diversos trabalhos, a Tabela 1 mostra uma serie de indicações de alvo de manejo, baseadas na IRFA ${ }_{95}$, destinadas as principais gramíneas forrageiras utilizadas em sistemas de produção intensiva de leite a pasto. Entretanto, o manejo do pastejo feito com base na IRFA 95 não é uma característica prática e fácil de ser adotada em situações de campo. Nesse sentido, diversos estudos foram feitos na tentativa de relacionar a altura com a IRFA ${ }_{95}$ (Freitas et al., 2012; Pedreira et al., 2007) embora a relação da altura com as características estruturais do dossel seja controversa, uma vez que um mesmo pasto pode atingir a IRFA 95 com diferentes alturas a depender de seu índice de área foliar residual ou do uso de adubação (Freitas et al., 2012). 
Como a interceptação luminosa tem demonstrado elevada correlação com a altura do dossel (Anjos et al., 2016; Carnevalli et al., 2006), esses dados são apresentados em termo de altura, uma vez que esta é uma variável prática de mais fácil adoção em condições de campo.

Tabela 1. Altura de entrada e saída de cultivares de gramíneas forrageiras indicadas para produção intensiva de leite a pasto.

\begin{tabular}{lcccc}
\hline Gênero & Cultivar & Entrada $(\mathrm{cm})$ & Saída $(\mathrm{cm})$ & Fontes \\
\hline \multirow{4}{*}{ Megathyrsus } & Mombaça & 90 & 50 & $\underline{\text { Euclides et al. (2018) }}$ \\
& Tanzânia & 70 & 35 & $\underline{\text { Euclides et al. (2014) }}$ \\
& BRS Zuri & $70-75$ & $35-40$ & $\underline{\text { Embrapa (2014) }}$ \\
& BRS Quênia & 70 & 35 & $\underline{\text { Jank et al. (2017) }}$ \\
Uruchloa & Marandu & 35 & $20-25$ & $\underline{\text { Anjos et al. (2016) }}$ \\
& Xaraés & 30 & 15 & $\underline{\text { Pedreira et al. (2009) }}$ \\
Cynodon & BRS Paiaguás & 65 & $30-35$ & $\underline{\text { Jaguaribe et al. (2019) }}$ \\
Pennisetum al. (2019) & $\underline{\text { Pedreira et al. (2007) }}$ \\
& BRS Ipyporã & 40 & 10 & $\underline{\text { Voltolini et al. (2010) }}$ \\
\hline
\end{tabular}

Fonte: Adaptado de diversos autores.

\section{Produção de leite}

Enquanto a produção de leite por animal é reflexo da qualidade da forragem ingerida, a produtividade por hectare é resultante do número de animais por área, ou seja, da produção de forragem (Gomide et al., 2012; Gomide et al., 2015). Voltolini et al. (2010) avaliaram duas frequências de pastejo, sendo uma com intervalo fixo de 26 dias e a outra variável conforme a IRFA 95 , em pastos de capim-elefante (Pennisetum purpureum Schum. cv. Cameroon). Não foram observadas diferenças na produção de leite e na composição do leite entre as diferentes frequências de pastejo. Porém a taxa de lotação foi $30 \%$ e a produção de leite $34 \%$ maior na área com frequência de pastejo variável conforme a IRFA95.

Trabalhos realizados com o capim-marandu também têm evidenciado a superioridade da adoção do período de descanso variável, conforme a IRFA ${ }_{95}$, em relação ao período de descanso com dias fixos (Anjos et al., 2016). Moura et al. (2017) avaliaram o reflexo das frequências de pastejo em pastos de capim-marandu, forrageira que compõe grande parte das áreas de pasto no país. Os autores observaram produção por área de 16 a $28 \%$ maior para pastos de capim-marandu submetidos ao período de descanso variável conforme a IRFA 95 em relação ao período de descanso fixo de 30 dias.

A maior produção por área em sistemas com adoção do período de descanso variável, conforme a IRFA $_{95}$, está relacionada ao menor período de descanso, tornando menor o número de piquetes necessários para a produção de leite a pasto. Para o capim-marandu, o intervalo entre pastejo com base na IRFA $_{95}$ tem proporcionado redução média de seis dias no intervalo entre pastejo, em relação ao período de descanso fixo de 30 dias (Moura et al., 2017). Resultados semelhantes foram observados em pastos de capim-elefante e capim-tanzânia. Voltolini et al. (2010) observaram intervalo médio de 19,4 dias para pastos de capim-elefante com período de descanso variável em relação ao período de descanso fixo de 26 dias, enquanto Chambela Neto et al. (2015) obtiveram redução no período de descanso de três a seis dias para o capim-tanzânia em comparação ao período de descanso fixo de 30 dias.

Resultados de pesquisas também têm evidenciado diferenças entre cultivares da mesma espécie, apesar destes serem submetidos a idênticas condições de manejo. Freitas (2019) avaliou por dois anos consecutivos a produção de leite de vacas mestiças Holandês x Zebu em pastos de BRS Zuri e BRS Quênia submetidos ao período de descanso variável conforme a IRFA 95 e a intensidade de desfolha de $50 \%$ da altura de entrada dos animais nos piquetes. Embora não tenha sido observada diferença na produção de massa de forragem no pré-pastejo, diferença na produção de leite por área foi observada entre os cultivares no segundo ano de avaliação, com o cultivar BRS Zuri superando o BRS Quênia em 15,6 $\mathrm{L} \mathrm{ha}^{-1}\left(129,6\right.$ vs. 114,0 $\left.\mathrm{L} \mathrm{ha}^{-1} \mathrm{dia}^{-1}\right)$. Esse fato é decorrente da maior capacidade de lotação do BRS Quênia, o que permitiu o referido acréscimo na produção por área. Conforme evidenciado por Fariña et 
al. (2011), o aumento na taxa de lotação promove reflexos positivos na produtividade, sendo mais eficiente do que a produção individual atribuída a incrementos em função do mérito genético.

\section{Conclusão}

A utilização de estratégias de manejo do pastejo, baseadas na morfofisiologia das plantas forrageiras tem se mostrado mais adequadas para o controle das características estruturais do dossel forrageiro, garantindo maior proporção de folhas e propiciando melhor condição para maximização do consumo em sistemas de pastejo rotativo. Nesse sentido, a utilização de estratégias de manejo com base nas alturas pré e pós-pastejo, podem favorecer sua utilização prática em condições de campo, auxiliando no desenvolvimento da atividade leiteira e aumentando os coeficientes de produtividade e consequentemente a rentabilidade do sistema.

O surgimento de novos cultivares de gramíneas forrageiras tem gerado a necessidade de desenvolver estratégias de manejo do pastejo que resultem na máxima eficiência produtiva de tais gramíneas, de modo a favorecer a incorporação das mesmas no sistema produtivo, garantindo a diversificação e sustentabilidade produtiva ao longo do tempo. Entretanto, o desenvolvimento de tais práticas trata-se de processo demorado e deve romper as barreiras acadêmicas, de modo a adentrar a porteira do produtor, pois só assim o processo de desenvolvimento obterá o êxito necessário.

\section{Referências bibliográficas}

Anjos, A. J., Gomide, C. A. M., Ribeiro, K. G., Madeiro, A. S., Morenz, M. J. F., \& Paciullo, D. S. C. (2016). Forage mass and morphological composition of Marandu palisade grass pasture under rest periods. Ciência e Agrotecnologia, 40(1), 76-86. https://doi.org/10.1590/S1413-70542016000100007

Araújo, S. A. C., Santos, C. C. R., Alves, D. A., Braz, T. G. S., Barroso, A. L., \& Dobbss, L. B. (2020). Grazing management strategies for Xaraés grass (Brachiaria brizantha cv. Xaraés). Archivos de Zootecnia, 69(265), 22-29.

Assis, A. G., Stock, L. A., Campos, O. F., Gomes, A. T., Zoccal, R., \& Silva, M. R. (2005). Sistemas de produção de leite no Brasil. Embrapa Gado de Leite-Circular Técnica, 1, 1-6.

Aurélio, N. D., Quadros, F. L. F., Maixner, A. R., Rossi, G. E., Daniel, E., Roman, J., Bandinelli, D. G., Trindade, J. P. P., \& Brum, M. S. (2007). Comportamento ingestivo de vacas holandesas em lactação em pastagens de capim-elefante anão (Pennisetum purpureum cv. Mott) e Tifton 85 (Cynodon dactylon x C. nlemfuensis) na região noroeste do Estado do Rio Grande do Sul. Ciência Rural, 37(2), 470-475. https://doi.org/10.1590/s0103-84782007000200027.

Barcellos, A. O., Ramos, A. K. B., Vilela, L., Junior, M., \& Bueno, G. (2008). Sustentabilidade da produção animal baseada em pastagens consorciadas e no emprego de leguminosas exclusivas, na forma de banco de proteína, nos trópicos brasileiros. Revista Brasileira de Zootecnia, 37(Supl.), 5167. http://dx.doi.org/10.1590/S1516-35982008001300008

Blaser, R. E. (1988). Pasture-animal management to evaluate plants and to develop forage systems. Simpósio Sobre Manejo da Pastagem, 9, 1-39.

Braga, G. J., Pedreira, C. G. S., Herling, V. R., \& Luz, P. H. D. C. (2007). Eficiência de pastejo de capim-marandu submetido a diferentes ofertas de forragem. Pesquisa Agropecuaria Brasileira, 42(11), 1641-1649. https://doi.org/10.1590/S0100-204X2007001100017

Cândido, M. J. D., Silva, R. G., Neiva, J. N. M., Facó, O., Benevides, Y. I., \& Farias, S. F. (2006). Fluxo de biomassa em capim-tanzânia pastejado por ovinos sob três períodos de descanso. Revista Brasileira de Zootecnia, 35(6), 2234-2242. https://doi.org/10.1590/S1516-35982006000800006.

Carnevalli, R. A., Silva, S. C., Bueno, A. A., Uebele, M. C., Bueno, F. O., Hodgson, J., Silva, G. N., \& Morais, J. P. G. (2006). Herbage production and grazing losses in Panicum maximum cv. Mombaça under four grazing managements. Tropical Grasslands, 40(3), 165.

Cecato, U., Machado, A. O., Martins, E. N., Pereira, L. A. F., Barbosa, M. A. A., \& Santos, G. T. (2000). Avaliação da produção e de algumas características da rebrota de cultivares e acessos de Panicum maximum Jacq. sob duas alturas de corte. Revista Brasileira de Zootecnia, 29(3), 660-668. https://doi.org/10.1590/S1516-35982000000300004 
Chambela Neto, A., Silva, J. F. C., Deresz, F., Fernandes, A. M., Deminicis, B. B., Haddade, I. R., \& Rosado, T. L. (2015). Morphology, morphogenesis and production of Tanzânia grass, submitted to either fixed or variable resting period. American Journal of Plant Sciences, 6(1), 163-171. https://doi.org/10.4236/ajps.2015.61019.

Clipes, R. C., Silva, J. F. C., Detmann, E., \& Vásquez, H. M. (2006). Composição químicobromatológica da forragem durante o período de ocupação em pastagens de capim-elefante (Pennisetum purpureum, Schum) e capim-mombaça (Panicum maximum, Jacq) sob manejo rotacionado. Arquivo Brasileiro de Medicina Veterinária e Zootecnia, 58(5), 868-876. https://doi.org/10.1590/S0102-09352006000500024.

Corsi, M. (1985). Potencial das pastagens para produção de leite. In A. M. Peixoto, J. C. Moura, \& V. P. Faria (Eds.), Bovinocultura leiteira: Fundamentos da exploração racional. (pp. 147-154).

Cóser, A. C., Martins, C. E., Fonseca, D. M., Salgado, L. T., Alvim, M. J., \& Teixeira, F. V. (1999). Efeito de diferentes períodos de ocupação da pastagem de capim-elefante sobre a produção de leite. Pesquisa Agropecuária Brasileira, 34(5), 861-866. https://doi.org/10.1590/s0100-204x1999000500018.

Embrapa. (2014). BRS Zuri, produção e resistência para a pecuária. Campo Grande, MS: Embrapa Gado de Corte. Disponível em: https://www.embrapa.br/documents/1355008/1528459/Folder+Zuri.pdf/e89a784d-fe75-47ff-8a796065f85b8fb5. Acesso em: 16 jul. 2019.

Euclides, V P B, \& Barbosa, R. A. (2008). Manejo de pastagem. In J. R. Verginassi \& M. V Fonseca (Eds.), Curso de formação, recuperação e manejo de pastagem. EMBRAPA.

Euclides, V P B, Carpejani, G. C., Montagner, D. B., Nascimento Junior, D., Barbosa, R. A., \& Difante, G. S. (2018). Maintaining post-grazing sward height of Panicum maximum (cv. Mombaça) at $50 \mathrm{~cm}$ led to higher animal performance compared with post-grazing height of $30 \mathrm{~cm}$. Grass and Forage Science, 73(1), 174-182. https://doi.org/10.1111/gfs.12292

Euclides, V. P. B., Macedo, M. C. M.,Valle, C. B., Barbosa, R. A., \& Gonçalves, W. V. (2008). Produção de forragem e características da estrutura do dossel de cultivares de Brachiaria brizantha sob pastejo. Pesquisa Agropecuária Brasileira, 43(12), 1805-1812. https://doi.org/10.1590/S0100-204X2008001200023

Euclides, V. P. B., Montagner, D. B., Difante, G. dos S., Barbosa, R. A., \& Fernandes, W. S. (2014). Sward structure and livestock performance in guinea grass cv: Tanzania pastures managed by rotational stocking strategies. Scientia Agricola, 71(6), 451-457. https://doi.org/10.1590/0103-90162013-0272.

Fariña, S. R., Garcia, S. C., Fulkerson, W. J., \& Barchia, I. M. (2011). Pasture-based dairy farm systems increasing milk production through stocking rate or milk yield per cow: pasture and animal responses. Grass and Forage Science, 66(3), 316-332. https://doi.org/10.1111/j.1365-2494.2011.00795.x.

Fonseca, D. M., Salgado, L. T., Queiroz, D. S., Cóser, A. C., Martins, C. E., \& Bonjour, S. C. M. (1998). Produção de leite em pastagem de capim-elefante sob diferentes períodos de ocupação dos piquetes. Revista Brasileira de Zootecnia, 27(5), 848-856.

Fonseca, D. M., Santos, E. R., \& Martuscello, J. A. (2010). Importância das forrageiras no sistema de produção. In D. M. Fonseca \& J. A. Martuscello (Eds.), Plantas forrageiras. Editora da Universidade Federal de Viçosa.

Freitas, F. P., Fonseca, D. M., Santos Braz, T. G., Martuscello, J. A., \& Santos, M. E. R. (2012). Forage yield and nutritive value of Tanzania grass under nitrogen supplies and plant densities. Revista Brasileira de Zootecnia, 41(4), 864-872. https://doi.org/10.1590/S1516-35982012000400006

Freitas, C. A. S. (2019) Valor nutritivo, produção de leite e dinâmica de serrapilheira em pastos de Megathyrsus maximus. 2019. 99 f. Tese (Doutorado em Zootecnia) - Universidade Federal de Viçosa, Viçosa, 2019

Gomide, C A M, Paciullo, D. S. C., Morenz, M. J. F., Deresz, F., \& Lopes, F. C. F. (2012). Potential of tropical forages for milk production on pasture. Informe Agropecuario, 33(266), 80-91.

Gomide, Carlos A M, Chaves, C. S., Ribeiro, K. G., Sollenberger, L. E., Paciullo, D. S. C., Pereira, T. P., \& Morenz, M. J. F. (2015). Structural traits of elephant grass (Pennisetum purpureum Schum.) genotypes under rotational stocking strategies. African Journal of Range \& Forage Science, 32(1), 
51-57. https://doi.org/10.2989/10220119.2014.930929.

Gomide, J. A. (1998). Fatores da produção de leite a pasto. Congresso Nacional de Estudantes de Zoootecnia, 1-32.

Gomide, J. A., Cândido, M. J. D., \& Alexabdrino, E. (2003). As interfaces solo-planta-animal da exploração da pastagem. As Interfaces Solo-Planta-Animal da Exploração da Pastagem, 4, 75-116.

Jaguaribe, T. L., Soares, N. A., Rodrigues, P. R., Bellan, J., Paciullo, D. S. C., Morenz, M. J. F., \& Gomide, C. A. de M. (2019). Estrutura do dossel, massa de forragem e produção de leite em pastagem de cultivares de Brachiaria sp. Embrapa Gado de Leite-Artigo Em Anais de Congresso.

Jank, L., Andrade, C. M. S., Barbosa, R. A., Macedo, M. C. M., Valério, J. R., Verzignassi, J. R., Zimmer, A. H., Fernandes, C. D., Santos, M. F., \& Resende, R. M. S. (2017). O capim-BRS Quênia (Panicum maximum Jacq.) na diversificação e intensificação das pastagens. Embrapa AcreComunicado Técnico, 1, 1-18.

Lima, J. B. M. P., \& Rodriguez, M. N. (2009). Manejo do pastejo de forrageiras tropicais. Carderno Técnico de Veterinária e Zootecnia, 60, 69-84.

Lopes, C. M., Paciullo, D. S. C., Araújo, S. A. C., Gomide, C. A. M., Morenz, M. J. F., \& Villela, S. D. J. (2017). Massa de forragem, composição morfológica e valor nutritivo de capim-braquiária submetido a níveis de sombreamento e fertilização. Arquivo Brasileiro de Medicina Veterinária e Zootecnia, 69(1), 225-233. http://dx.doi.org/10.1590/1678-4162-9201.

Lopes, F. C. F. (2008). Consumo de forrageiras tropicais por vacas em lactação sob pastejo em sistemas intensivos de produção de leite. Cadernos Técnicos de Veterinária e Zootecnia, 57, 67-117.

Martha Jr., G. B.; Barioni, L. G.; Vilela, L. \& Barcellos, A. O. (2003). Área de Piquete e taxa de lotação no pastejo rotacionado. Embrapa Planaltina - Comunicado Técnico, 101, 1-8

Moura, A. M., Tomich, T. R., Pereira, L. G. R., Teixeira, A. M., Paciullo, D. S. C., Jayme, D. G., Machado, F. S., Gomide, C. A. M., Campos, M. M., \& Chaves, A. V. (2017). Pasture productivity and quality of Urochloa brizantha cultivar Marandu evaluated at two grazing intervals and their impact on milk production. Animal Production Science, 57(7), 1384-1391. https://doi.org/10.1071/AN16715.

Paciullo, D. S. C., Gomide, J. A., Silva, E. A. M., Queiroz, D. S., \& Gomide, C. A. M. (2002). Características anatômicas da lâmina foliar e do colmo de gramíneas forrageiras tropicais, em função do nível de inserção no perfilho, da idade e da estação de crescimento. Revista Brasileira de Zootecnia, 31(2), 890-899. http://dx.doi.org/10.1590/S1516-35982002000400012

Pedreira, B. C., Pedreira, C. G. S., \& Silva, C. S. (2007). Estrutura do dossel e acúmulo de forragem de Brachiaria brizantha cultivar Xaraés em resposta a estratégias de pastejo. Pesquisa Agropecuária Brasileira, 42(2), 281-287. http://dx.doi.org/10.1590/S0100-204X2007000200018

Pedreira, B., Pedreira, C. G. S., \& Silva, S. C. (2009). Acúmulo de forragem durante a rebrotação de capim-xaraés submetido a três estratégias de desfolhação. Revista Brasileira de Zootecnia, 38(4), 618-625. http://dx.doi.org/10.1590/S1516-35982009000400005

Pereira, A. V, \& Cóser, A. C. (2002). Forrageiras para corte e pastejo. Embrapa Gado de Leite-Circular Técnica, 1, 219-244.

Pinto, J. C., \& Ávila, C. L. S. (2013). Terminologia e classificação de plantas forrageiras. In R A Reis, T. F. Bernardes, \& G. R. Siqueira (Eds.), Forragicultura: Ciência, tecnologia e gestão de recursos forrageiros. Gráfica Multipress.

Pinto, L. F. M., Silva, S. C., Sbrissia, A. F., Augusto, C., Carvalho, B., Carnevalli, R. A., Fagundes, J. L., \& Pedreira, C. G. S. (2001). Dinâmica do acúmulo de matéria seca em pastagens de Tifton 85 sob pastejo. Scientia Agricola, 58(3), 439-447. http://dx.doi.org/10.1590/S0103-90162001000300001

Pompeu, R., Cândido, M. J. D., Neiva, J. N. M., Rogério, M. C. P., Benevides, Y. I., \& Oliveira, B. C. M. (2009). Fluxo de biomassa em capim-tanzânia sob lotação rotativa com quatro níveis de suplementação concentrada. Revista Brasileira de Zootecnia, 38(05), 809-817. http://dx.doi.org/10.1590/S1516$\underline{35982009000500005}$

Porto, P. P., Deresz, F., Santos, G. T., Lopes, F. C. F., Cecato, U., \& Cóser, A. C. (2009). Milk production, milk composition, intake and digestibility of tropical forages under intermittent grazing system. Revista Brasileira de Zootecnia, 38(8), 1422-1431. https://doi.org/10.1590/S1516- 


\section{5}

Reis, R. A., Ruggieri, A. C., Casagrande, D. R., \& Páscoa, A. G. (2009). Suplementação da dieta de bovinos de corte como estratégia do manejo das pastagens. Revista Brasileira de Zootecnia, 38(spe), 147-159. https://doi.org/10.1590/S1516-35982009001300016

Rigolon, L. P., Do Prado, I. N., Cavalieri, F. L. B., Do Nascimento, W. G., \& Negrão, J. A. (2003). Effect of different levels of energy intake on production and viability of embryos in heifers and cows. Revista Brasileira de Zootecnia, 32(6). https://doi.org/10.1590/S1516-35982003000600004.

Santos, F. A. P., Martinez, J. C., Greco, L. F., Carareto, R., \& Penati, M. A. (2008). Nutrição de vacas em lactação, no período chuvoso, para a produção intensiva de leite em pasto. Caderrnos Técnicas Veterinária e Zootecnia, 57, 1-39.

Santos, W. B. R., Santos, G. T., Silva-Kazama, D. C., Cecato, U., Marchi, F. E., Visentainer, J. V, \& Petit, H. V. (2011). Production performance and milk composition of grazing dairy cows fed pelleted or non-pelleted concentrates treated with or without lignosulfonate and containing ground sunflower seeds. Animal Feed Science and Technology, 169(3-4), 167-175. https://doi.org/10.1016/j.anifeedsci.2011.06.015

Schmitt, D., Padilha, D. A., Dias, K. M., Santos, G. T., Rodolfo, G. R., Zanini, G. D., \& Sbrissia, A. F. (2019). Chemical composition of two warm-season perennial grasses subjected to proportions of defoliation. Grassland Science, 65(3), 171-178. https://doi.org/10.1111/grs.12236

Silva, J. J., Carvalho, D. M. G., Gomes, R. A. B., \& Rodrigues, A. B. C. (2012). Produção de leite de animais criados em pastos no Brasil. Veterinária e Zootecnia, 17(1), 26-36.

Teixeira, R. M. A., \& Oliveira, A. S. (2009). Manejo de vacas leiteiras em pastejo. In J. C. P. M. Silva, A. S. Oliveira, \& C. M. Veloso (Eds.), Manejo e administração na bovinocultura leiteira. Universidade Federal de Viçosa Imprensa Universitaria.

Van Soest, P. J. (1994). Nutritional Ecology of the Ruminant. In Nutritional Ecology of the Ruminant (Vol. 1, Issue 2). Cornell University Press. https://doi.org/10.7591/9781501732355

Voltolini, T. V., Santos, F. A. P., Martinez, J. C., Clarindo, R. L., Penati, M. A., \& Imaizumi, H. (2010). Características produtivas e qualitativas do capim-elefante pastejado em intervalo fixo ou variável de acordo com a interceptação da radiação fotossinteticamente ativa. Revista Brasileira de Zootecnia, 39(5), 10021010. https://doi.org/10.1590/S1516-35982010000500009

Zanini, G. D., Santos, G. T., \& Sbrissia, A. F. (2012). Frequencies and intensities of defoliation in Aruana guineagrass swards: Morphogenetic and structural characteristics. Revista Brasileira de Zootecnia, 41(8), 1848-1857. https://doi.org/10.1590/S1516-35982012000800007

Recebido: 16 de junho, 2020

Aprovado: 22 de agosto, 2020

Disponível online: 23 de outubro, 2020
Licenciamento: Este artigo é publicado na modalidade Acesso Aberto sob a licença Creative Commons Atribuição 4.0 (CC-BY 4.0), a qual permite uso irrestrito, distribuição, reprodução em qualquer meio, desde que o autor e a fonte sejam devidamente creditados. 